\title{
Schreibcoaching und Schreibberatung - der Versuch einer Abgrenzung
}

\author{
Eva Kuntschner ${ }^{1}$ (D) \\ Angenommen: 10. Dezember 2020 / Online publiziert: 30. Dezember 2020 \\ (c) Der/die Autor(en) 2020
}

\section{Zusammenfassung}

Klickt man sich durch die Websiten von Kolleg*innen ,aus der Schreibszene“, findet man viele unterschiedliche Namen für deren Angebote: Schreibtraining, Schreibwerkstätten, Schreibworkshops, Schreibberatung, Schreibcoaching ... Dabei wirkt es so, also ob sich Schreibtraining, Schreibwerkstätten und Schreibworkshops eher auf die Arbeit mit Gruppen beziehen, während Schreibberatung und Schreibcoaching für Einzelsettings gebräuchlicher zu sein scheinen. Der Unterschied bzw. die Ähnlichkeit von Schreibcoaching und Schreibberatung bleibt dabei oftmals unklar, was nicht zuletzt bei den Ratsuchenden (aber auch bei den Anbieter*innen selbst) für Verwirrung sorgen kann.

Der vorliegende Artikel beschäftigt sich mit den beiden letztgenannten Begriffen „Schreibcoaching“ und „Schreibberatung“ und versucht diese einer näheren Begriffsbestimmung und -abgrenzung zuzuführen. Dies soll dazu beitragen, Unklarheiten in diesem Bereich zu beseitigen und Schreibberater*innen und Schreibcoaches in der begrifflichen Verortung ihrer Angebote mehr Sicherheit zu bieten.

Schlüsselwörter Schreibberatung $\cdot$ Schreibcoaching $\cdot$ Begriffsbestimmung $\cdot$ Beratungsprozess

\section{Writing Counseling, Writing Coaching}

\begin{abstract}
Clicking through the websites of collegues "in the writing community" you can find many different names for the products they offer: writing training, writing workshops, writing counseling, writing coaching ... This that writing training and writing workshops are targeted at groups, while writing counseling and writing coaching are used for one-on-one setting. The differences and/or similarities between writing counseling and writing coaching remain often unclear in this respect, which in turn is cause for confusion among potential clients and service providers alike.

This article discusses the two terms "writing coaching" and "writing counseling" and attemps to define and differentiate them in more detail. The aim is to contribute to removing uncertainties in this regard and to provide service providers with a surer footing in the description of the products they offer.
\end{abstract}

Keywords Writing counseling $\cdot$ Writing coaching $\cdot$ Definition $\cdot$ Counseling process

Information zu den Abbildungen Die Abbildungen wurden mit PowerPoint erstellt.

Eva Kuntschner

eva.kuntschner@univie.ac.at, kuntschner@gmail.com

1 Center for Teaching and Learning, Universität Wien,

Universitätsstraße 5, 1010 Wien, Österreich 


\section{Einleitung}

Vor kurzem erreichte mich ein E-Mail eines potenziellen Kunden, der auf der Suche nach Unterstützung in seinem Dissertationsprozess war. Er schrieb ${ }^{1}$ :

Guten Tag!

Ich kontaktiere Sie auf Empfehlung von [einer Kollegin von Ihnen] bezüglich Unterstützung im Schreibprozess - oder derzeit besser: „Nichtschreib“-Prozess - meiner Dissertation. [Ihre Kollegin] meinte, Sie wären die richtige Ansprechpartnerin, ,wenn es um ein echtes Coaching und nicht um eine Schreibberatung geht“". Obwohl ich gestehen muss, dass mir der Unterschied noch nicht ganz klar ist [Hervorhebung d. Verf.]. Vielleicht können wir alles Weitere in den nächsten Tagen einmal telefonisch besprechen? [...] Herzlichen Dank vorab, [Name]

Dieses E-Mail brachte mich zum Nachdenken, weshalb ich es zum Anlass nahm, mich genauer mit dem Unterschied zwischen Schreibberatung und Schreibcoaching zu beschäftigen. Auf die Erkenntnisse aus dieser Beschäftigung möchte ich in diesem Text eingehen, da sie meiner Meinung nach einem besseren Verständnis von Schreibcoaching als eigenem Format dienen, das sich klar abgrenzbar von anderen unterscheidet. Ich bin mir sicher, dass vieles, was ich in diesem Artikel beschreibe, im konkreten Tun von Schreibcoaches schon zur Anwendung kommt. Mit diesem Artikel möchte ich vor allem einen Beitrag dazu leisten, dieses konkrete professionelle Handeln zu systematisieren und theoretisch zu untermauern. Ein genaueres Verständnis der Gemeinsamkeiten und Unterschiede dieser beiden Formate kann meiner Meinung nach dazu beitragen, die Angebote von Schreibberater*innen und Schreibcoaches zu schärfen und diesbezüglich existierende Unsicherheiten, von denen Kolleg*innen immer wieder berichten und die ich auch an mir selbst festgestellt habe, etwas zu mildern.

Nach meinem Kenntnisstand wird die Frage nach dem, was Schreibberatung bzw. Schreibcoaching eigentlich ist, in der Literatur nicht besonders in den Fokus genommen. Dies hängt vielleicht mit der grundsätzlichen Schwammigkeit des Begriffs „Beratung“ einerseits und dem oftmalig inflationären Gebrauch des Begriffs „Coaching“ andererseits zusammen (so zumindest meine Hypothese). Auch in Bezug auf diese Unschärfen versuche ich in den folgenden Abschnitten einen Beitrag zur Klärung zu leisten.

${ }^{1}$ Ich habe das E-Mail anonymisiert; die Veröffentlichung erfolgt mit Einverständnis des Verfassers.

\section{Schreibcoaching und Schreibberatung - der Versuch einer Abgrenzung}

Jemand wie der Verfasser des oben zitierten E-Mails, der auf der Suche nach Unterstützung in einem schwierigen Schreibprozess ist, hält sich für gewöhnlich nicht mit konzeptuellen Überlegungen zur Unterscheidung zwischen verschiedenen Beratungsformaten auf. Doch für Berater*innen, die mit Schreibenden arbeiten, kann die Beschäftigung mit den Unterschieden zwischen den einzelnen Formaten durchaus fruchtbar und dadurch im Endeffekt auch für ihre Ratsuchenden, Kund*innen, Klient*innen oder Coachees hilfreich sein. Denn was ist nun wirklich der Unterschied zwischen Schreibberatung und Schreibcoaching?

Beginnen wir mit einer Gemeinsamkeit: Sowohl bei Schreibberatung als auch bei Schreibcoaching handelt es sich um Formen der Beratung. Doch was bedeutet dies? Ein Blick in die Literatur zeigt, dass die Verunsicherung des Verfassers des E-Mails diesbezüglich als durchaus repräsentativ gelten kann. So schreibt Kallmeyer (2000, S. 228):

Die Begriffe „Beraten“/,Beratung“ kennzeichnen einen Gegenstandsbereich mit unscharfen Grenzen und unterschiedlichen Benennungen. [...] Die Unschärfe der Gegenstandsabgrenzung liegt an der Verwandtschaft von Beraten mit anderen Handlungsformen der Hilfe oder der Unterstützung. So kann Beraten stärker auf Auskunftgeben und Wissensvermittlung oder stärker auf die Thematisierung und Beeinflussung von psychischen Befindlichkeiten orientiert sein. In manchen Kontexten ist ein wichtiger Bestandteil des Beratungskonzepts, gerade keine Ratschläge zu geben, sondern nur die Lösungssuche des Klienten [sic] zu unterstützen.

Dieses Zitat zeigt sehr gut, dass die fließenden Grenzen zwischen unterschiedlichen Beratungsformen die Abgrenzung zueinander extrem schwierig gestaltet und nicht nur in Bezug auf den Themenkreis „Schreiben“ zu Verwirrung führt.

Um dieser Verwirrung klärend entgegenzutreten, bleibe ich noch etwas bei den Gemeinsamkeiten zwischen Schreibcoaching und Schreibberatung: Beide Formate beschäftigen sich mit dem Feld „Schreiben“. Dieses ist weit gefächert und erstreckt sich vom wissenschaftlichen über berufliches und hin zum kreativen Schreiben. Mögliche Themengebiete für Beratung sind hier einerseits die unterschiedlichen Genres, die es zu meistern gilt, also z.B. die wissenschaftliche Abschlussarbeit, das innerbetriebliche Memo oder auch das Sonett. Andererseits beschäftigt sich v.a. die prozessorientierte Schreibberatung auch mit dem Schreibprozess und seinen Herausforderungen, die in den 
unterschiedlichen Stadien einer Textproduktion entstehen. Diese Aspekte kommen, zumindest meiner Erfahrung nach, in jeder Form der Beratung zum Thema „Schreiben“ vor, sei es nun Schreibberatung oder Schreibcoaching.

Wenn nun das Themenfeld von Schreibberatung und Schreibcoaching dasselbe ist, so muss der Unterschied zwischen den beiden Formaten wohl woanders zu finden sein: Der Blick in die Literatur zeigt, dass die meisten Expert*innen nach Unterschieden im Fokus verschiedener Beratungsformen differenzieren (vgl. dazu z. B. Nestmann 2007b). Schreibberatung ist eine bestimmte Form der Fachberatung, die den Ratsuchenden fachliches Know-How zum Themengebiet „Schreiben“ bietet. Dies geht aus der folgenden Definition von Schreibberatung von Grieshammer et al. (2013, S. IX) klar hervor: „Wir begreifen Schreibberatung als freiwilliges Unterstützungsangebot für Studierende (und in weiteren Kontexten allgemein für Schreibende), das Schreibenden einen geschützten Raum für den konzentrierten Austausch über ihr Schreibprojekt sowie für ihre Fragen und Sorgen bietet." Dies bedeutet also, dass es in Schreibberatung vorrangig um das Schreibprojekt und die damit einhergehenden Fragen und Probleme geht - also um Fachinhalte. Aus diesem Grund halte ich an dieser Stelle die Definition von Fachberatung von Wimmer et al. (2012, S. 10) für hilfreich. Sie schreiben:

Fachberatung verstehen wir somit als einen gewollten und gesteuerten Kommunikationsprozess zwischen einem oder mehreren Beraterinnen und Beratern und einem oder mehreren Klienten [und Klientinnen] mit dem Ziel,

- durch Vermitteln von sachlichen Informationen und fachlichem Wissen

- den Klientinnen Grundlagen für Entscheidungen und Handlungsmöglichkeiten zu geben,

- um ihre Situation, ihre Anliegen und ihre Probleme verändern, lösen oder bewältigen zu können.

Sowohl Grieshammer et al. als auch Wimmer et al. sehen demnach den Fokus von Fachberatung auf der Vermittlung von sachlichen Informationen und fachlichem Wissen, durch die die Klient*innen bei der Lösungsfindung unterstützt werden. Überträgt man dies nun auf die Schreibberatung, dann ergibt sich daraus, dass diese Form der Beratung Schreibende durch die Vermittlung von fachlichem Wissen in Bezug auf den Schreibprozess bzw. das dabei angestrebte Produkt unterstützt. Dies deckt sich mit Dörings Definition, die in einem Blogeintrag zum Thema Schreibberatung folgende Punkte anführt:

1. Schreibberatung ist eine freiwillige, kurzfristige, oft nur situative, soziale Interaktion zwischen Ratsuchendem und Schreibberater.
2. Schreibberatung verfolgt das Ziel, im Beratungsprozess eine Entscheidungshilfe zur Bewältigung eines von dem/der Ratsuchenden vorgegebenen aktuellen Schreibproblems zu geben.

3. Schreibberatung erreicht dieses Ziel durch Vermittlung von Informationen und/oder Einüben von Fertigkeiten gemeinsam mit dem/der Ratsuchenden. (2017)

Schreibberatung als Fachberatung fokussiert also darauf, den (möglicherweise problematischen) Schreibprozess einer ratsuchenden Person durch die punktuelle, lösungsorientierte und auf das konkrete Schreibproblem bezogene Vermittlung von Fachinhalten zum Themenfeld „Schreiben" zu unterstützen.

Der Fokus von Coaching liegt im Vergleich dazu woanders. Anders als bei Fachberatung geht es nicht primär um die Vermittlung von Fachinhalten und unterstützenden Fertigkeiten. Laut Astrid Schreyögg, einer der führenden Coaching-Theoretiker*innen im deutschen Sprachraum, geht es beim Coaching als Maßnahme der „Personalentwicklung“2 um die ,Funktionsfähigkeit von [Coachees] in ihrem beruflichen Umfeld“" (Schreyögg 2009/2012, S. 203). Sie schreibt: „Dabei geht es zunächst ohne Ansehen des einzelnen Menschen um die Mitarbeiterschaft [in] einer Organisation, die qua Arbeitsvertrag verpflichtet ist, die Ziele des organisatorischen Systems möglichst optimal zu erreichen.“

Ausgehend vom Führungskräfte-Coaching sehen Kern und Stadler (2010, S. 202) das Hauptaugenmerk von Coaching hauptsächlich auf die rollenspezifischen Aspekte einer bestimmten Aufgabe gerichtet. Angewandt auf Schreibcoaching liegt der Fokus also auf der Frage, wie eine Person in ihrer Rolle als Schreibende*r ihre Aufgabe bestmöglich erfüllen und damit ihrer Rolle gerecht werden kann.

Dieser zielorientierte Fokus auf die rollenspezifische Erfüllung einer bestimmten Arbeitsaufgabe lässt sich mit dem Ursprung des Wortes „Coaching“ näher erklären, der im Transportwesen liegt. Thierau-Brunner und Stelzer-Rothe (2018, S. 7) zitieren Renton, die schreibt:

(...) the noun "Coach" derives from a "large kind of carriage", usually of the grander variety, initially built from the mid-15th century onwards in the Hungarian town of Kocs (pronounced "coach"). The word spread throughout the rest of Europe and perhaps the analogy that can be drawn with current executive coaches is that they helped get you from A to B, preferably in the quickest possible time (...) (vgl. Renton, J. 2009, S. 2).

\footnotetext{
${ }^{2}$ Im Gegensatz zu Supervision, die sie als „Personen-Entwicklung“ bezeichnet (Schreyögg 2009/2012, S. 203).
} 


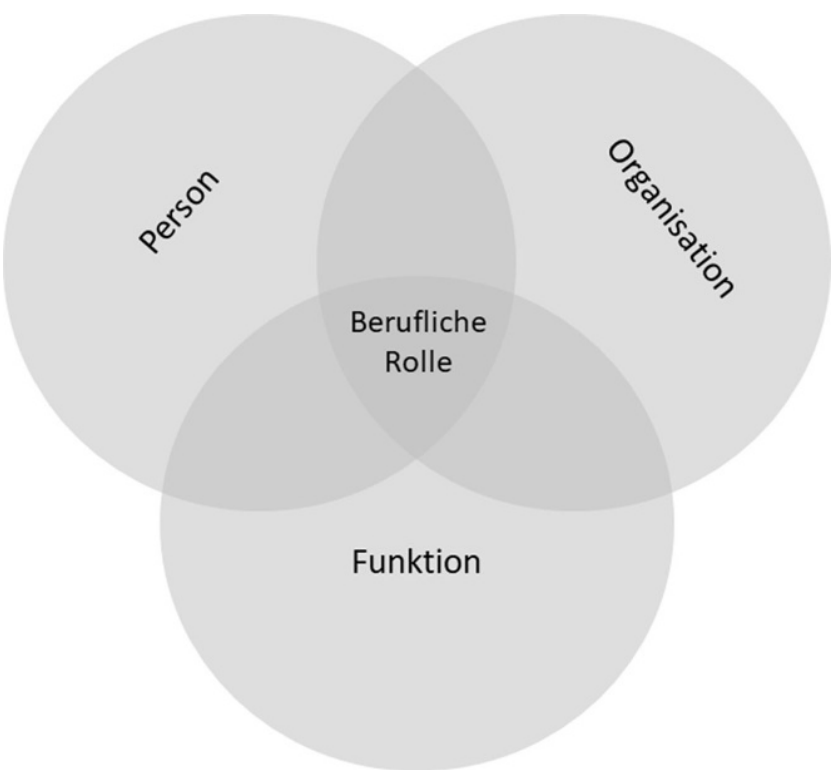

Abb. 1 Die berufliche Rolle als Schnittmenge zwischen Person - Organisation - Funktion

Aus diesem Zitat wird der Fokus von Coaching auf Rolle ganz klar ersichtlich: Es geht darum, eine Aufgabe (in dem hier zitierten Beispiel: Transport von A nach B) so gut wie möglich zu erfüllen und damit der eigenen beruflichen bzw. aufgabenorientierten Rolle bestmöglich gerecht zu werden.

Der Rollen-Diskurs nimmt in den Sozial- und Bildungswissenschaften und in der Psychoanalyse seit langem viel Raum ein und würde den Rahmen dieses Artikels um ein Vielfaches sprengen (vgl. dazu z.B. Dahrendorf 1968). Coaching als Form der „berufsbezogenen Beratung“" (Schreyögg 2009/2012, S. 196) fokussiert auf die „berufliche Rolle [als] Schnittmenge aus dem, was eine Organisation von einer Person erwartet (und bereit ist, zu geben) und dem, was die Person von der Organisation erwartet (und bereit ist zu geben)“. Dabei wird „ein Teil der Rolle ... durch die Stellenbeschreibung definiert: Ziele, Aufgaben und Befugnisse der Stelle“ (ibid.). Anders gesagt (und wie in Abb. 1 dargestellt): Bei der beruflichen Rolle handelt es sich um die Schnittmenge zwischen Person, Organisation und Funktion.

Obwohl es formalisierte Rahmenbedingungen wie Arbeitsverträge in Bezug auf Schreibprojekte meistens nicht gibt, so werden diese doch oft im Kontext einer „Mitarbeiterschaft in einer Organisation“ (ibid.) durchgeführt (z.B. in einer bestimmten Firma oder an einer Universität). Die „Funktion“, in der Arbeitswelt aus der Stellenausschreibung ersichtlich, findet sich beim Schreiben oftmals in der Definition des Schreibprojekts (,Dissertant*in“, z.B.). Aus der Schnittmenge dieser beiden Aspekte mit der Person, die das Schreibprojekt durchführt, ergibt sich also die für das Schreibcoaching relevante Rolle der "Schreibenden“.

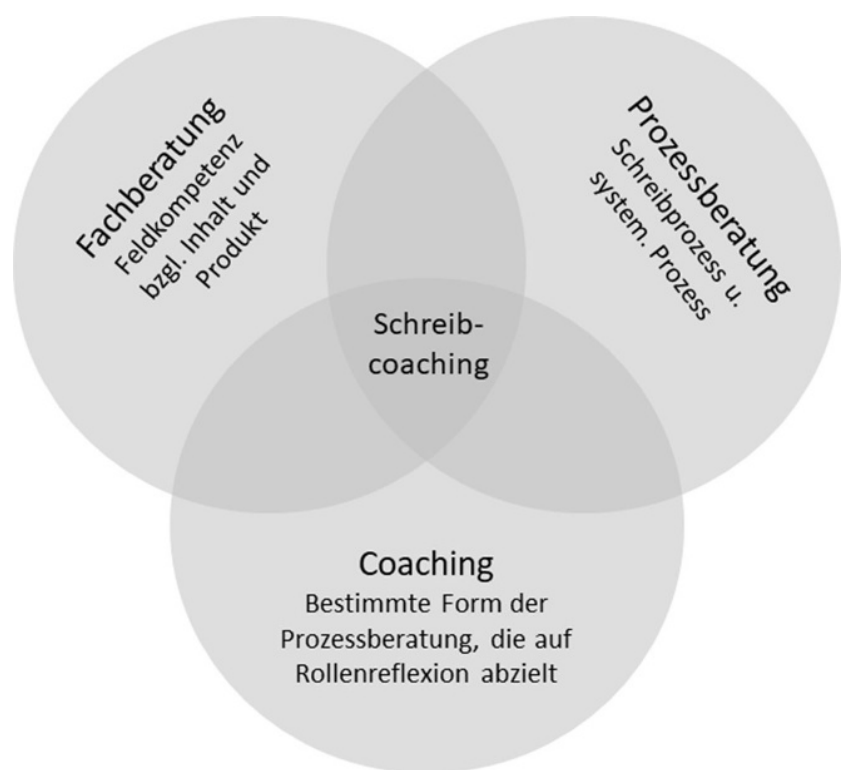

Abb. 2 Schreibcoaching als Schnittmenge von Fachberatung, Prozessberatung und Coaching

Für das Schreibcoaching bedeutet dies nun: Eine Schreibcoach unterstützt die Coachee ${ }^{3}$ dabei, das Schreibprojekt bestmöglich durchzuführen, indem die Coach die Coachee in der Reflexion und fallweisen Optimierung der Schnittmenge aus Person, Organisation und Funktion unterstützt. Um dies tun zu können, benötigt die Coach unbedingt Feldkompetenz (Kern und Stadler 2010, S. 202), also Wissen über Produkte und Zusammenhänge des Schreibprozesses. Darüber hinaus benötigt sie aber noch spezielles Wissen und Kompetenzen im Bereich der Rollenreflexion und der Gestaltung des Coachingprozesses. Das Zusammenspiel dieser unterschiedlichen Elemente wird aus der Grafik in Abb. 2 ersichtlich:

Aus dieser Abbildung wird deutlich, dass es für Fachberatung Feldkompetenz in Bezug auf Inhalt und Produktwissen braucht. Für Prozessberatung benötigt man wiederum Know-How zum Schreibprozess einerseits und generelles systemisches Prozesswissen andererseits. Coaching als dritte Komponente wiederum ist eine bestimmte Form der Prozessberatung, die es zum Ziel hat, die Rolle als „Schreibende*r" zu reflektieren (Beispiele siehe etwas weiter unten im Text).

Daraus folgt: Während Schreibberatung als spezielle Form der Fachberatung (mit höherer oder geringerer Prozessorientierung, je nach beraterischem Ansatz) sich mit

\footnotetext{
${ }^{3}$ Ich werde in Folge die Begriffe „Schreibcoach“ und „Schreibcoachee" im generischen Femininum verwenden. Damit trage ich einerseits der besseren Lesbarkeit Rechnung und andererseits der Tatsache, dass es sich sowohl bei den Beratenden als auch bei den Ratsuchenden in diesem Feld sehr oft um Frauen* handelt. Männer* sind in beiden Fällen selbstverständlich mitgemeint.
} 
der zu erfüllenden Schreibaufgabe beschäftigt, so fokussiert Schreibcoaching zusätzlich zum Prozess des Schreibens stark auf die Reflexion der Rolle der Schreibenden. Daraus ergibt sich demnach folgender Unterschied zwischen Schreib-Fachberatung und Schreibcoaching: Schreibberatung hat vornehmlich einen Aufgaben- und Sachfokus, während Schreibcoaching hauptsächlich einen Rollen- und Personenfokus hat.

Durch die Fokussierung auf die Person der Schreibenden werden im Schreibcoaching also auch Aspekte des Prozesses in den Blick genommen, die bei einem reinen Sachbzw. Aufgabenfokus außer Acht gelassen werden (können): Gefühle, Un- bzw. Vorbewusstes, Unverdautes, Widersprüche und Ambivalentes. Dies geht über Fachberatung hinaus und fordert von der Coach Kompetenzen, die fachliches Know-How zum Thema „Schreiben“ übersteigen und die ich im nächsten Abschnitt detaillierter betrachte.

\section{Die für "Schreibcoaching" nötigen Kompetenzen}

Um die für Schreibcoaching nötigen Kompetenzen genauer zu bestimmen, erscheint mir eine Betrachtung der Art der Tätigkeit, die Schreibcoaching darstellt, als hilfreich. Schreibcoaching kann als eine Form der „Beziehungsarbeit" (vgl. Nestmann 2007a) bezeichnet werden. Dazu schreiben Busse und Tietel (2018, S. 43):

... Beziehungsarbeiter müssen Arbeitsbündnisse mit Klienten, Patienten, Mandanten und Schutzbefohlenen etc. eingehen. Das Besondere daran ist, dass die Person(en) und der Arbeitsgegenstand partiell zusammenfallen. Das heißt, dass ein Arbeitsbündnis z.B. zwischen Helfer und Klient zur co-konstruktiven Bearbeitung eines Anliegens (z.B. einer lebensweltlichen Krise) geschlossen werden muss.

Umgelegt auf Schreibcoaching bedeutet dies, dass in dieser Beratungsform ein Arbeitsbündnis zwischen Schreibcoach und Schreibcoachee zur co-konstruktiven Bearbeitung eines Anliegens (z.B. einer Schreibkrise) geschlossen werden muss. Weiter oben habe ich dargestellt, warum im Sinne der Unterscheidung zwischen Schreib-Fachberatung und Schreibcoaching die Bearbeitung dieses Anliegens nur teilweise durch die Vermittlung von Fachinhalten erfüllt werden kann. Denn im Schreibcoaching geht es darüber hinaus einerseits um den verstärkten Blick auf die Person bzw. die Rolle der Schreibcoachee, andererseits um die im Rahmen eines Schreibprojekts entstehenden Spannungsfelder. $\mathrm{Zu}$ diesen zählen:
- Spannungsfelder im inneren Schreibprozess:

- Schreibende ${ }^{*} r$-äußere Anforderungen - innere Wünsche: Wie weit muss man sich äußeren Regeln wie z. B. Genreanforderungen beugen, wie weit kann man seine eigenen Wünsche und Vorstellungen realisieren?

- Schreibende $*_{r}$ - Ängste - Wünsche: Was passiert, wenn der Text gut wird? Wem komme ich dann in die Quere? Über wen erhöhe ich mich? Was motiviert, was hindert mich?

- Spannungsfelder im äußeren Schreibprozess:

- Schreibende*r - Schreibprozess - Schreibprodukt (inkl. Genreanforderungen, Deadlines etc.): Wie kann der Schreibprozess so gestaltet werden, dass er einerseits der Person der Schreibenden, andererseits den Anforderungen an das Endprodukt gerecht wird?

- Schreibende ${ }^{*} r$-Anforderungen des Schreibprojekts andere lebens- und arbeitsweltiche Anforderungen: Diese Aspekte tauchen im Coachingprozess oftmals als Fragen zum ,Zeitmanagement“ oder zu „Selbstoptimierung " auf (und sind in dieser Form von der Coach durchaus zu hinterfragen): Wie kann ich schneller werden beim Lesen, beim Schreiben? Wie bringe ich die vielen verschiedenen beruflichen und privaten Anforderungen an mich ,unter einen Hut"?

Ein weiterer wichtiger Aspekt, der im Schreibcoaching eine große Rolle spielt, ist das, was Busse und Tietel (2018, S. 30) als ,institutionelle Rahmenbedingungen (Gesetze, Normen, Regeln) und gesellschaftlich-institutionelle Aufträge an Organisationen“ bezeichnen. Es gilt, die Normen und Regeln des jeweilig zu erfüllenden Genres zu beachten und, z. B. im Falle von wissenschaftlichen Qualifizierungsarbeiten, sich an Plagiatsgesetze zu halten, deren Übertretung gravierende Konsequenzen nach sich ziehen kann. Doch auch andere, implizite Normen und Regeln spielen beim Schreiben immer eine große Rolle:

„Versteht man, was ich hier sagen möchte?“

„Ist das ein Gedicht?“،

„Passt das so?““

„Lehne ich mich hier zu weit hinaus, wenn ich schreibe ...?"

„Beleidige ich in dieser Passage jemanden?“

Mit diesen und ähnlichen Fragen werden sich Coachees im Verlauf eines Coachingprozesses häufig an die Schreibcoach. Die Angst, diese Regeln und Normen nicht zu erfüllen bzw. zu verletzen, ist oftmals fast körperlich im Beratungsraum spürbar und steht immer im Spannungsfeld mit dem Bedürfnis, sich selbst auszudrücken und verständ- 
lich zu machen ${ }^{4}$. Hier ist es daher sehr wichtig, dass die Schreibcoach die Fähigkeit besitzt, diese Spannung auzubalancieren und nicht „auf eine Seite zu kippen“ - denn das könnte entweder zu große Rigidität und Regelkonformität oder aber zu verständnisvolles oder schmeichelndes Feedback nach sich ziehen, was beides der Schreibcoachee keinen Dienst erweisen würde.

Die Aufgabe der Schreibcoach besteht demnach in all diesen Fällen darin, ,ausgleichend und ausbalancierend mal parteiergreifend und stützend, ein anderes Mal in kritische Distanz gehend“ (Busse und Tietel 2018, S. 45) zu handeln. Dies geht über die reine Vermittlung von Fachinhalten hinaus, wodurch sich dieser Aspekt von Schreibcoaching meiner Meinung nach klar von Schreib-Fachberatung abgrenzen lässt.

\section{Zusammenschau}

Was bedeuten diese Überlegungen also nun in Hinblick auf die Frage aus dem in der Einleitung zitierten E-Mail? Wie könnte meine Antwort aussehen? Ein Vorschlag wäre:

Sehr geehrter Herr [Name], vielen Dank für Ihr E-Mail und die spannende Frage zu Schreibcoaching, die mich zum Nachdenken über die Unterschiede und die daraus folgende $\mathrm{Ab}$ grenzung zwischen den Formaten "Schreibberatung“ und "Schreibcoaching“ angeregt hat. In meinem Verständnis ist Schreibberatung eine bestimmte Form der Fach-Beratung und fokussiert auf die Fachinhalte und auf den Schreibprozess. Schreibcoaching hingegen fügt dem noch den Aspekt der Prozessberatung hinzu, die auf Rollenreflexion abzielt. Im Schreibcoaching geht es also darum, die Coachees in ihrer Rolle als Schreibende zu begleiten und zu unterstützen.

Soweit eine Zusammenfassung meiner Überlegungen zu der von Ihnen aufgeworfenen Frage, die ich hoffentlich im Ansatz beantworten konnte. Natürlich gäbe es zu diesem spannenden und komplexen Thema noch Vieles zu sagen. Ich freue mich auf eine anregende und angeregte Diskussion!

Mit herzlichem Dank für den Denkanstoß und freundlichen Grüßen,

Eva Kuntschner

\footnotetext{
${ }^{4}$ Dies wird auch durch Forschungen zu den Affekten Schreibender bestätigt. Siehe hierzu z. B. Sondra Perl (1980). Sie schreibt: „,The move is not to any words on the page nor to the topic but to feelings or nonverbalized perceptions that surround the words, or to what the words already present evoke in the writer" (364-365, Hervorhebung im Original).
}

P.S.: Die konkrete Anfrage bezüglich Schreibcoaching für Ihre Dissertation beantworte ich in einem weiteren E-Mail.

Danksagung Ich bedanke mich bei Dr.in Adelheid Wimmer, Kerstin Löffler, MSc, und Mag.a Hannah Steiner, BA.

Interessenkonflikt E. Kuntschner gibt an, dass kein Interessenkonflikt besteht.

Open Access Dieser Artikel wird unter der Creative Commons Namensnennung 4.0 International Lizenz veröffentlicht, welche die Nutzung, Vervielfältigung, Bearbeitung, Verbreitung und Wiedergabe in jeglichem Medium und Format erlaubt, sofern Sie den/die ursprünglichen Autor(en) und die Quelle ordnungsgemäß nennen, einen Link zur Creative Commons Lizenz beifügen und angeben, ob Änderungen vorgenommen wurden.

Die in diesem Artikel enthaltenen Bilder und sonstiges Drittmaterial unterliegen ebenfalls der genannten Creative Commons Lizenz, sofern sich aus der Abbildungslegende nichts anderes ergibt. Sofern das betreffende Material nicht unter der genannten Creative Commons Lizenz steht und die betreffende Handlung nicht nach gesetzlichen Vorschriften erlaubt ist, ist für die oben aufgeführten Weiterverwendungen des Materials die Einwilligung des jeweiligen Rechteinhabers einzuholen.

Weitere Details zur Lizenz entnehmen Sie bitte der Lizenzinformation auf http://creativecommons.org/licenses/by/4.0/deed.de.

\section{Literatur}

Busse, S., \& Tietel, E. (2018). Mit dem Dritten sieht man besser: Triaden und Triangulierung in der Beratung. Göttingen: Vandenhoeck \& Ruprecht.

Dahrendorf, R. (1968). Homo Sociologicus. Ein Versuch zur Geschichte, Bedeutung und Kritik der Kategorie der sozialen Rolle (7. Aufl.). Köln: Westdeutscher Verlag.

Döring, K. (2017). Wie kann man Schreibberatung definieren und wie lässt sie sich in der Beratungspsychologie verorten? Blogeintrag. https://openblog.hypotheses.org/199 (Erstellt: 5. Sept. 2017). Zugegriffen: 26. Juni 2020.

Grieshammer, E., Liebetanz, F., Peters, N., \& Zegenhagen, J. (2013). Zukunftsmodell Schreibberatung. Eine Anleitung zur Begleitung von Schreibenden im Studium. Baltmannsweiler: Schneider Hohengehren.

Kallmeyer, W. (2000). Beraten und Betreuen: Zur gesprächsanalytischen Untersuchung von helfenden Interaktionen. Zeitschrift für qualitative Bildungs-, Beratungs- und Sozialforschung, 1(2000), 227-252. URN: http://nbnresolving.de/urn:nbn:de:0168-ssoar280593.

Kern, S., \& Stadler, C. (2010). Psychodrama. Eine Einführung. Wiesbaden: Springer VS.

Nestmann, F. (2007a). Beratung zwischen alltäglicher Hilfe und Profession. In F. Nestmann (Hrsg.), Disziplinen und Zugänge. Das Handbuch der Beratung, (Bd. 1, S. 547-558). Tübingen: DGVTVerlag.

Nestmann, F. (Hrsg.). (2007b). Disziplinen und Zugänge. Das Handbuch der Beratung, Bd. 1. Tübingen: DGVT-Verlag.

Schreyögg, A. (2012). Besonderheiten des Coaching - Unterschiede zur Supervision. In H. Pühl (Hrsg.), Grundlagen - Praxis - Perspektiven. Handbuch der Supervision, (Bd. 3, S. 196-208). Berlin: Ulrich Leutner. (2009/2012).

Thierau-Brunner, H., \& Stelzer-Rothe, T. (2018). Coaching hat viele lebendige Facetten. In T. Stelzer-Rothe \& H. Thierau-Brunner 
(Hrsg.), Einblicke in systemisches Coaching. Theorie, Praxisfälle, Erfahrungen. Berlin:: Wissenschafts-Verlag.

Wimmer, A., Wimmer, J., Buchacher, W., \& Kamp, G. (2012). Das

Beratungsgespräch: Skills und Tools für die Fachberatung. Wien:

Linde.

Publisher's Note Springer Nature remains neutral with regard to jurisdictional claims in published maps and institutional affiliations. 